\title{
Biosensing with T-ray spectroscopy
}

\author{
Bernd M. Fischer ${ }^{a, b}$, Hanspeter $\operatorname{Helm}^{b}$ and Derek Abbott ${ }^{a}$ \\ ${ }^{a}$ Centre for Biomedical Engineering and School of Electrical \& Electronic Engineering, The \\ University of Adelaide, Adelaide, SA 5005, Australia; \\ ${ }^{b}$ Department of Molecular and Optical Physics, Albert-Ludwigs-Universität Freiburg, D-79104 \\ Freiburg, Germany
}

\begin{abstract}
In the recent years, it has been shown that terahertz (or T-ray) spectroscopy is a versatile tool for biosensing and safety applications. This is due to the fact that the THz-spectra of many biomolecules show very characteristic, distinct spectroscopic features. Furthermore, most non-metallic packaging materials are nearly transparent in this frequency range $\left(0.1-6 \mathrm{THz}, 3 \mathrm{~cm}^{-1}-200 \mathrm{~cm}^{-1}\right)$, so that it is possible to non-invasively identify even sealed substances like pharmaceuticals, illicit drugs or explosives by their spectroscopic signatures. This opens a significant potential for a wide range of applications from quality control of pharmaceutical substances via safety applications through to biomedical applications.

The individual spectroscopic features below approximately $5 \mathrm{THz}$ that spurred the increased world wide interest in T-ray spectroscopy are mainly due to intermolecular rather than intramolecular vibrations in the polycrystalline samples. The spectra of more complex biomolecules, like proteins and nucleotides, typically show less or even no sharp features, due to the lack of long- range intermolecular order. Furthermore, due to the typically significantly smaller sample amount, the signal to noise ratio is strongly increased. Water shows a strong absorption in this frequency range, which all together makes real biomedical applications of T-ray spectroscopy rather difficult. Yet, by combining a careful sample preparation, novel experimental techniques and an advanced signal processing of the experimental data we can still clearly distinguish between even complex biomolecules and therefore demonstrate the potential the technique holds for biomedical applications.
\end{abstract}

Keywords: Terahertz time-domain spectroscopy, biomolecules, intermolecular vibrations, T-rays

\section{INTRODUCTION}

The terahertz (or T-ray) spectra of many small molecules of biomedical or pharmaceutical interest show very distinctive, characteristic features. These spectral signatures can be used to distinguish clearly between molecules of similar structure and even isomers. ${ }^{1}$ Combined with the fact that many packaging materials like polymers, paper, or even clothing are nearly transparent for terahertz radiation, this high sensitivity of T-ray spectra to minor changes in the molecular structure has spurred a world wide interest in the exploitation of this technique for applications ranging from pharmaceutical quality control ${ }^{2,3}$ to defence and security applications. ${ }^{4}$ In particular $\mathrm{THz}$ imaging, where each pixel of the 2-dimensional image contains the full $\mathrm{THz}$ spectral information, has proven to be a versatile tool for chemical recognition and biosensing. In the past few years, the increased interest in the field has led to new technical achievements in effective generation and detection of terahertz radiation and thus led to more compact and affordable systems. ${ }^{5} \quad$ However, many of the richly structured spectra of the smaller molecules originate rather from intermolecular than from intermolecular vibrations. Whereas this fact opens up a new potential for this technique to investigate and distinguish crystalline structure, ${ }^{6}$ it also means that when the samples lack a regular intermolecular structure, the corresponding spectra also lack the characteristic specific signatures. Most of these samples, such as for example proteins, ${ }^{7}$ RNA and DNA, therefore show quite unspecific, broad absorptions. However, in some cases it is still possible to use the absorption spectra to differentiate between such samples. ${ }^{8}$ This can still be very useful for biomedical applications where the focus is often not to identify a given substance but rather to differentiate between given substances.

Further author information: (Send correspondence to Bernd M. Fischer)

Bernd M. Fischer: E-mail: bernd.fischer@adelaide.edu.au, Telephone: +61 883034115

Derek Abbott: E-mail:dabbott@elecengadelaide.edu.au, Telephone: +61 883035748

Biophotonics 2007: Optics in Life Science, edited by Jürgen Popp, Gert von Bally, Proc. of SPIE-OSA Biomedical Optics, SPIE Vol. 6633, 66331D, (C) 2007 SPIE-OSA · 1605-7422/07/\$18 


\section{EXPERIMENTAL}

The $\mathrm{THz}$ spectra shown in this work have been recorded with different systems in Germany and Australia: Most of the spectra were recorded at the University of Freiburg/Germany using custom built THz time-domain spectrometer in transmission geometry. For some samples, a spectrometer in reflection geometry is more suitable. For these purposes, a custom built reflection spectrometer is available in Freiburg. The third spectrometer used in this work is a commercially available fibre-coupled THz spectrometer (T-ray 2000, Picometrix) which can be used either in transmission or reflection geometry. This system is located at the National T-ray Facility in Adelaide.

All THz time-domain spectrometers used in this study have in common that they are based on photoconductive antennas ${ }^{9}$ in order to generate and detect the $\mathrm{THz}$ pulses. The antennas of the custom built systems in Freiburg are made of low-temperature grown GaAs. The antenna geometry consists of two photolithographically deposited parallel metal $(\mathrm{Au} / \mathrm{Ge} / \mathrm{Ni})$ strip-lines, separated by several tens of micrometers. By illuminating the gap between the two metal lines the resulting free carriers are accelerated in the bias field and thus lead to the emission of a short electromagnetic pulse. The detector chip consists of a similar structure, yet no bias is applied on the metal lines. Only when the incoming $\mathrm{THz}$ pulse and the electromagnetic pulse temporally coincide at the detector, a current proportional to the field strength of the $\mathrm{THz}$ pulse can be detected. By varying the time delay between the $\mathrm{THz}$ pulse and the gating femtosecond laser pulse, the full temporal shape of the $\mathrm{THz}$ pulse can be recorded. The dielectric function of the material under investigation is determined in transmission THz-TDS by measuring a reference pulse $E_{\text {ref }}(t)$ propagating through an empty spectrometer and a sample pulse $E_{\text {sam }}(t)$ propagating through the spectrometer with the sample of thickness $d$ placed in the beam path. The field of the THz pulse transmitted through the sample $E_{\mathrm{sam}(\mathrm{t})}$ is modified by the frequency dependent index of refraction $n(\nu)$ and absorption $\alpha(\nu)$ of the sample. The two pulses are transformed into the frequency domain, and the amplitude $A(\nu)$ and phase $\Phi(\nu)$ of the ratio of the two spectra,

$$
\begin{aligned}
\frac{E_{\mathrm{sam}}(\nu)}{E_{\mathrm{ref}}(\nu)} & =\frac{4 n}{(n+1)^{2}} \exp [-\alpha d / 2+i 2 \pi \nu(n-1) d / c] \\
& =A(\nu) \exp [i \Phi(\nu)]
\end{aligned}
$$

are calculated and analyzed to obtain the spectra of the absorption coefficient

$$
\alpha(\nu)=-\frac{2}{d} \ln \left\{A(\nu) \frac{[n(\nu)+1]^{2}}{4 n(\nu)}\right\}
$$

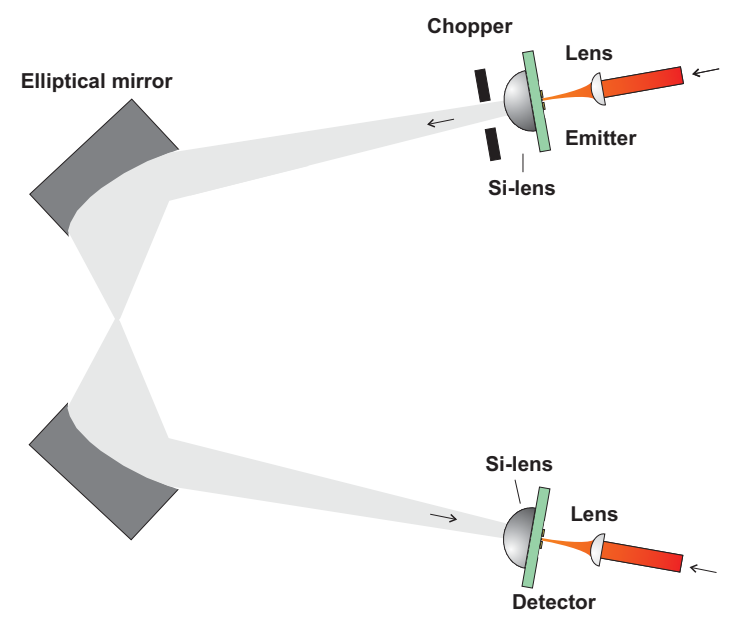

(a)

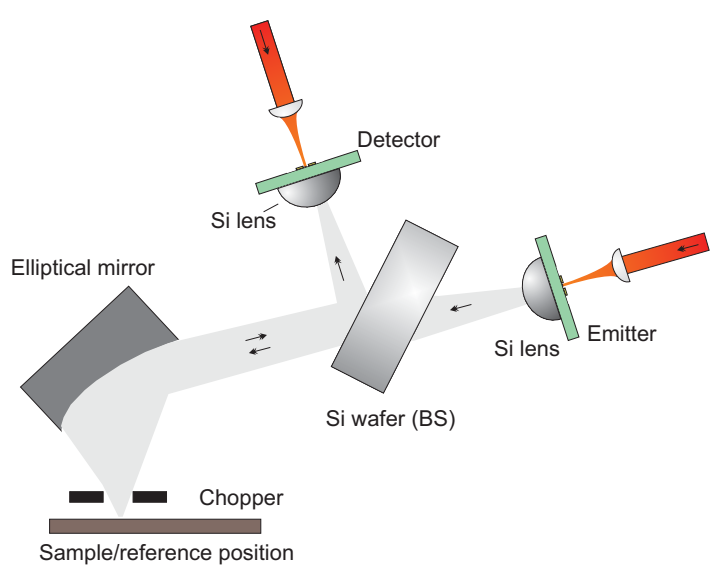

(b)

Figure 1. Schematic diagram of the custom built $\mathrm{THz}$ time-domain spectrometer in (a) transmission geometry and (b) in reflection geometry. 

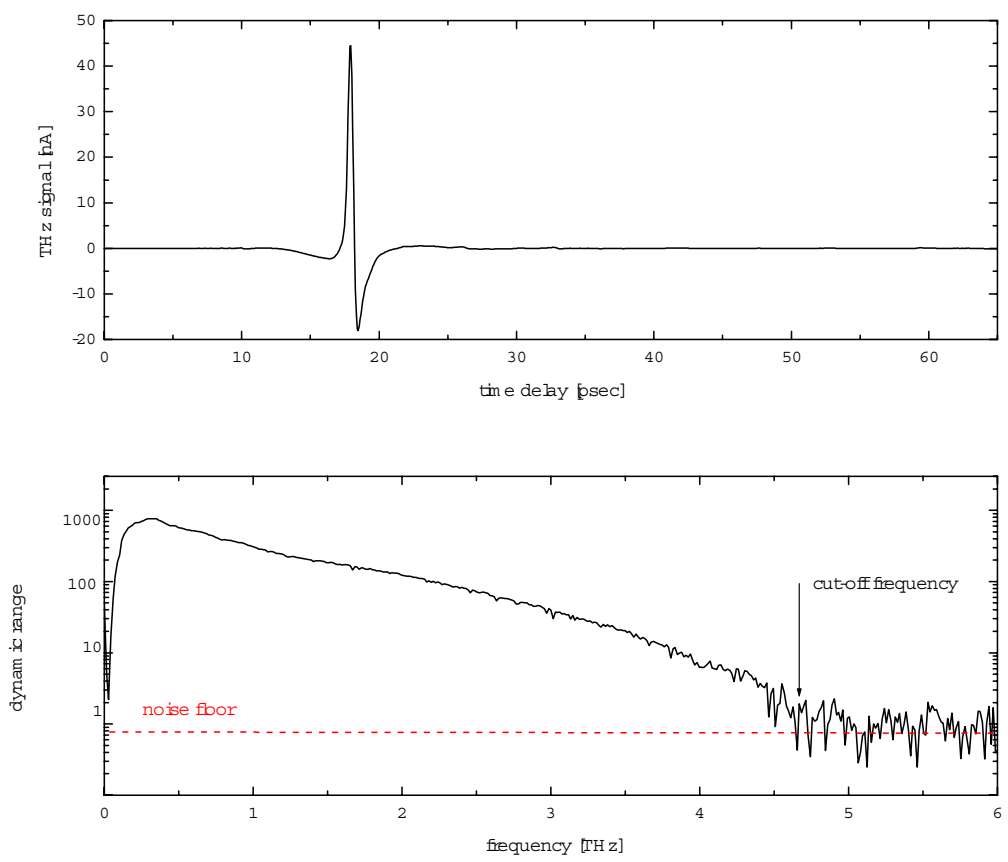

Figure 2. Typical $\mathrm{THz}$ pulse (upper curve) and corresponding frequency spectrum of the custom-built transmission time-domain spectrometer in Freiburg/Germany. The upper limit of the accessible frequency range is reached when the detected signal reaches that of the noise floor as indicated in the lower graph.

and index of refraction

$$
n(\nu)=1+\frac{c}{2 \pi \nu d} \Phi(\nu)
$$

In Figure 2, a typical $\mathrm{THz}$ pulse is shown together with its frequency spectrum. Owing to the typical singlecycle nature of the $\mathrm{THz}$ pulse, its frequency spectrum extends from the low gigahertz region to several THz. At high frequencies the spectrum is characterized by a gradual roll-off, until the detected signal level approaches that of the noise floor of the experiment.

For some samples a measurement in transmission geometry is not applicable. In particular water and other polar solvents show a very strong absorption in the far-infrared region. Also phonon-modes in ionic crystals show typically absorptions that exceed the limits given by the dynamic range of the experiment. ${ }^{10} \mathrm{~A}$ method to circumvent the limitations of THz-TDS, described above, is to operate the $\mathrm{THz}$ spectrometer in the reflection mode. In this case the reference signal typically consists of the THz pulse reflected from a silver mirror surface with a reflection coefficient close to minus unity. The sample signal consists of the $\mathrm{THz}$ pulse reflected from the plane sample surface, positioned at the same plane as the reference surface. Alternatively, the reference and sample signals are obtained as reflections from the front and the backside of a thick window in front of the sample. ${ }^{11}$ Here we consider only the case of normal incidence with a metal mirror as a reference surface. In the frequency domain the ratio between the sample and the reference spectra is then

$$
A(\nu) \exp [i \Phi(\nu)]=\frac{\hat{n}-1}{\hat{n}+1}=\frac{n+i \kappa-1}{n+i \kappa+1}
$$

Equation 4 can be inverted, and simple expressions for the index of refraction and absorption coefficient can be 
found:

$$
\begin{gathered}
n(\nu)=\frac{1-A(\nu)^{2}}{1+A(\nu)^{2}-2 A(\nu) \cos \Phi(\nu)}, \\
\alpha(\nu)=\frac{2 \pi \nu}{c} \frac{2 A(\nu) \sin \Phi(\nu)}{1+A(\nu)^{2}-2 A(\nu) \cos \Phi(\nu)} .
\end{gathered}
$$

In order to record the temperature dependence of the spectral features, the samples are mounted in a cryostat equipped with TPX windows. The temperature is measured near the sample by a calibrated Si-diode with an accuracy of $\pm 1 \mathrm{~K}$. The cryostat can be moved so that the $\mathrm{THz}$ beam passes through either the sample or through an empty aperture of the identical size as the clear aperture of the sample. By mounting the sample on a XYstage, a two dimensional image of the sample can be recorded, where each pixel consists of the full spectral information.

\section{RESULTS}

\subsection{Small molecules: chemical recognition and imaging}

Many small molecules of biomedical interest (like for example many pharmaceutical drugs) are at ambient temperature polycrystalline powders. It has been shown that the T-ray spectra of such samples typically are dominated by very distinct, characteristic features. These features are very sensitive to the molecular and crystalline structure of the biomolecular samples, and can therefore be used to identify different molecules and even different isomerization. ${ }^{1,12,13}$ As an example we compare in figure 3(a) the spectra of polycrystalline $\alpha$ and $\beta$-D-glucose, recorded at 10K. Despite the pronounced similarity of the molecular structure - differing only in the orientation of a single hydroxyl group on the anomeric carbon - a clear difference in the T-ray spectra is observed. In sharp contrast to these richly structured, characteristic spectra, the T-ray spectrum of a normal plastic bag (polyethylene) and a typical mail envelope is shown in figure 3(b). One can see that these typical packing materials show only low absorption, and in particular they show a featureless, broad slope that can be easily subtracted as background when the spectra of samples consisting of polycrystalline substances such as for example glucose inside such packing materials are recoded. This clearly shows the potential of this technique not only to differentiate between different samples but - once a database for a wide range of substances has been recorded - also to contact-free identify even concealed substances.

As the T-ray spectra of most pharmaceuticals, ${ }^{2,3,6}$ but also explosives and illicit drugs ${ }^{14}$ show such very characteristic signatures, $\mathrm{THz}$ time-domain spectroscopy not only allows for quality control of pharmaceuticals even through their polymer packaging, but also for safety and security applications and contact-free identification of illicit substances. In order to demonstrate this potential for chemical recognition of concealed samples, we show in figure4(a) a photograph of a sample consisting of polycrystalline lactose (1), acetylsalicylic acid (Aspirin) (2), sucrose (3), and tartaric acid (4), pressed into a polyethylene matrix for better stability. The four pellets were glued on paper, mounted a styrofoam plate and covered with a second layer of paper. The sample was then mounted on a 2-axis translation stage and moved in steps of $2 \mathrm{~mm}$ in order to record a two dimensional image. At each sample position a full $\mathrm{THz}$ pulse was recorded. Each pixel of this image therefore still contains the whole $\mathrm{THz}$ spectroscopic information, and by using signal processing and comparing the spectroscopic data with a database, the different substances can be recognized by their spectroscopic signatures. In figure 4(b) a false color image of the sample is shown. The spectrum recorded for each pixel was analyzed by comparing the spectral signatures with those of a spectroscopic database. In this preliminary study, only the most dominant signatures for each substance were used for comparison, and a color was assigned for a positive match. Despite the simplicity of this very basic and straightforward analysis, the four samples can already be clearly identified. It is obvious that by applying more complex and comprehensive signal processing the sensitivity can be significantly increased.

In some cases, a careful comparison of the spectra of molecules of very similar structure or even different isomers can help to identify the corresponding molecular vibrations that give rise to the characteristic absorptions in the T-ray spectra. ${ }^{1}$ However, in most cases these vibrations are typically of intermolecular rather than intramolecular character, and thus phonon-like vibrations of the crystalline structure. ${ }^{15,16}$ This can be easily demonstrated by comparing the spectra of the same sample in the crystalline or amorphous state respectively. ${ }^{15}$ 


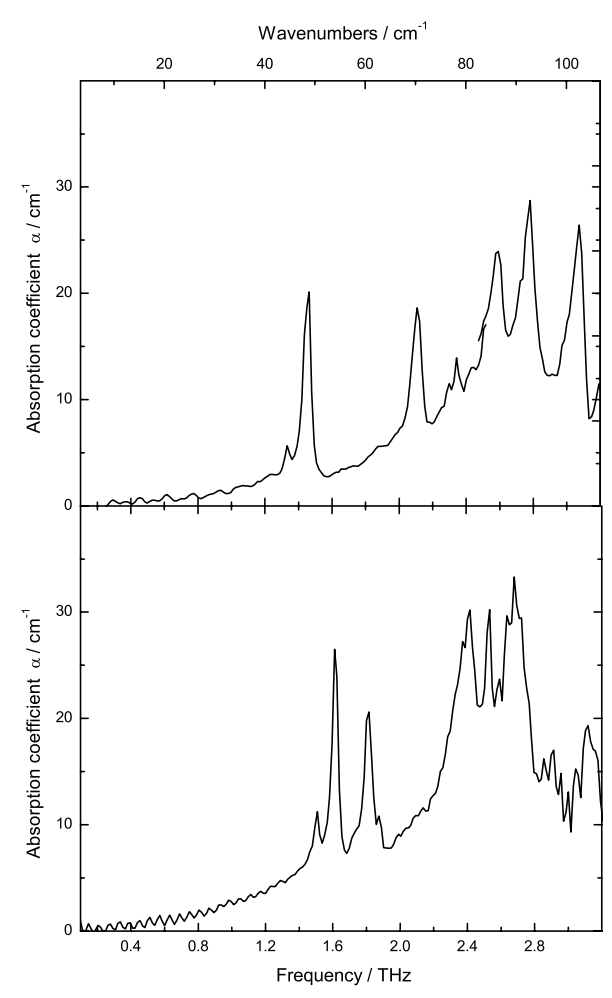

(a) .

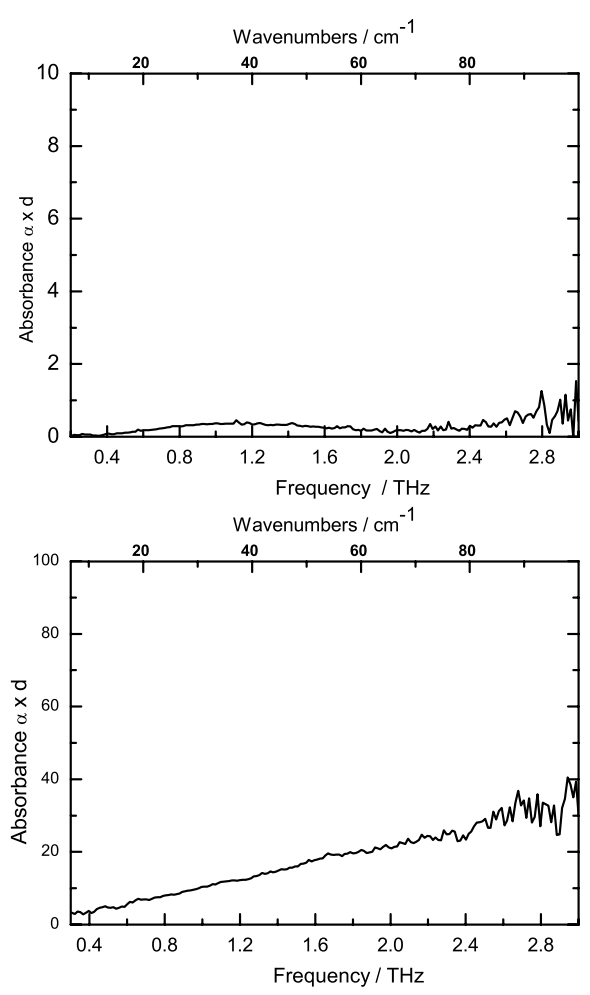

(b)

Figure 3. (a) Absorption coefficient $\alpha$ of $\alpha$-D-glucose (upper graph) and $\beta$-Glucose (lower graph) recorded at $10 \mathrm{~K}$. (b) Absorbance $\alpha \times d$ of typical packing materials.

We could also recently show that simulations of THz spectra based on DFT calculations only lead to satisfactory agreements between simulated and experimental spectra if intermolecular vibrations are considered. ${ }^{17}$ An easy experimental demonstration that clearly shows that the spectra of many polycrystalline samples are dominated by features arising from intermolecular vibrations is shown in figure 5, where we compare the T-ray spectra of a mixture of enantiopure Cystine and racemic Cystine. Both samples consist of an equal amount of D- and LCystine, yet whereas in the case of the mixture we mixed an equal amount of polycrystalline enantiopure Dand L-Cystine, in the racemic crystal, the two isomers are both constituents in the same crystal and arranged in a well-ordered, specific, alternating structure. ${ }^{18}$ Despite the fact that both samples consist of the same two enantiomer of Cystine, the spectra differ considerably from each other. As the only difference between the two samples is their intermolecular structure, this clearly demonstrates that the modes that give rise to the characteristic features in these spectra are intermolecular vibrations rather than purely intramolecular vibrations, as the latter would lead to the same signatures in the mixture of the pure enantiomers and the racemic sample. Whereas on the one hand this demonstrates the new potential for $\mathrm{THz}$ TDS spectroscopy to investigate the crystalline purity of pharmaceuticals ${ }^{6}$ and other crystalline samples, it also indicates that if the sample lacks a well ordered, intermolecular structure the $\mathrm{THz}$ spectra will show less or even no characteristic $\mathrm{THz}$ signatures.

\subsection{Large, complex bimolecules}

In contrast to the spectra of small, polycrystalline molecules, the $\mathrm{THz}$ spectra of most larger, more complex biomolecules typically show no distinct, characteristic features. Although theoretical simulations indicate a large number of normal modes in this frequency range, ${ }^{19}$ with typical THz TDS spectrometers these modes are most often not resolved, but a broad featureless absorption is observed. ${ }^{7,17}$ 


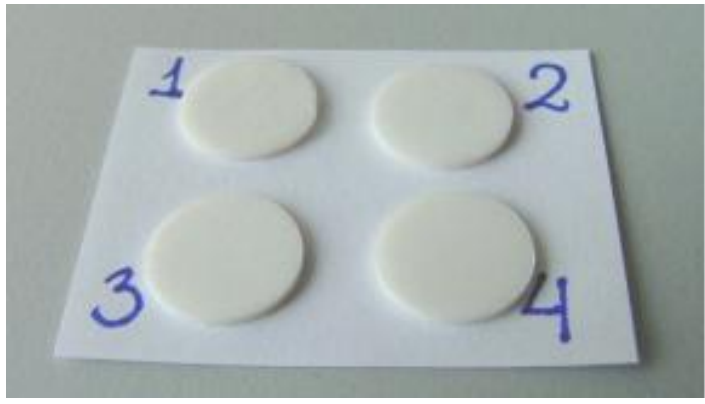

(a)

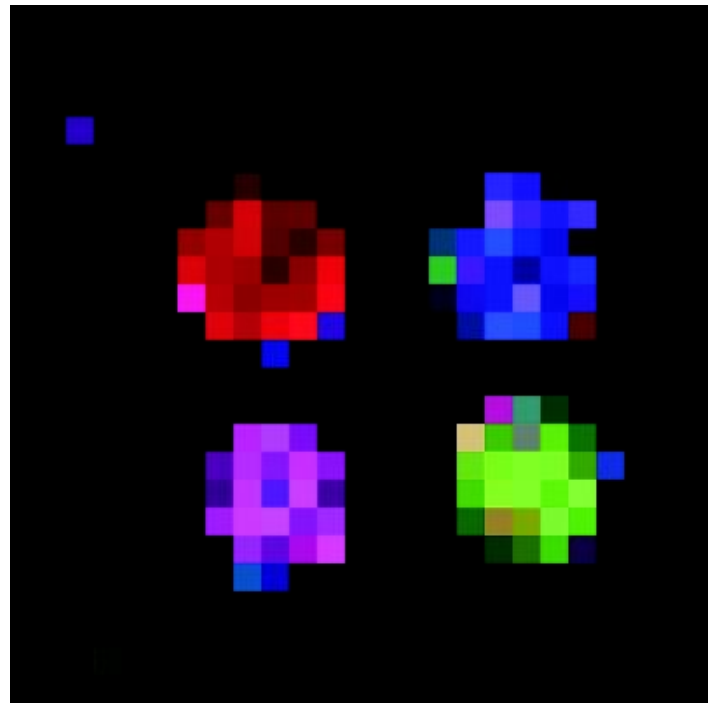

(b)

Figure 4. (a) Photograph of the sample consisting of four pellets of PE mixed with polycrystalline lactose (1), aspirin (2), sucrose (3), and tartaric acid (4). The pellets were glued on a sheet of paper, mounted on a styrofoam plate and covered with a second layer of paper. (b) False color image of the sample. Each of the four colors represents a spectroscopic signature that is characteristic for one of the samples respectively. Each of the samples can thus be clearly identified through the styrofoam and paper packing layers.

Despite the drawback induced by the lack of characteristic features in the spectra of such larger, complex biomoleules, THz-TDS can still be a versatile tool for biosensing. Recently, Nagel et al. have shown that by loading a planar $\mathrm{THz}$ resonator with the sample material, the binding state of DNA an be determined. Experiments have indicated that the modification of the effective dielectric load leads to a measurable shift of the resonance frequency. ${ }^{20}$ However, these techniques require the use of specially designed resonator structures and

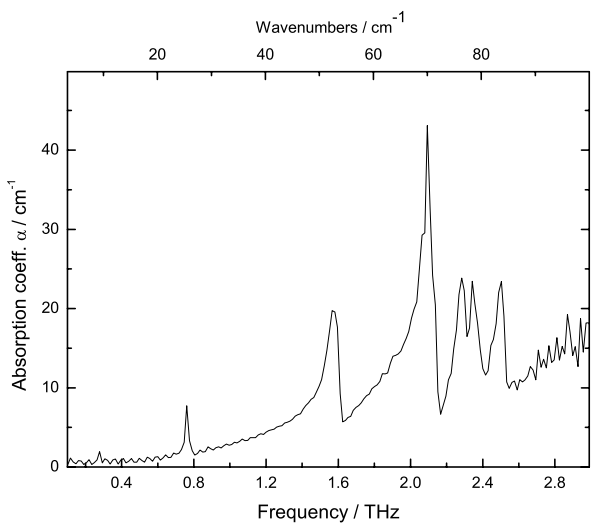

(a)

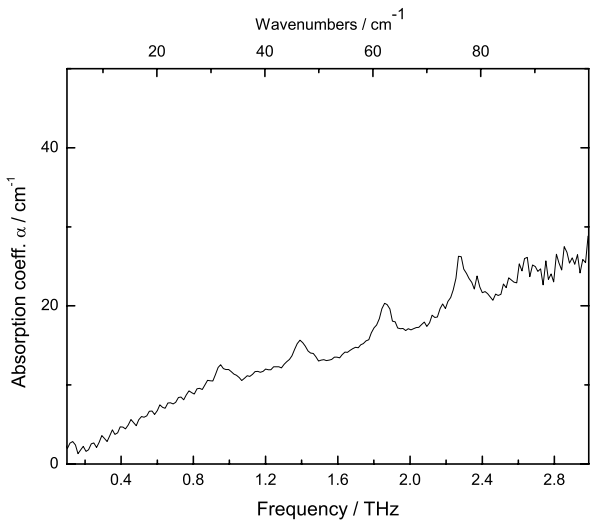

(b)

Figure 5. Terahertz absorption spectrum of (a) a mixture of polycrystalline enantiopure D- and L- Cystine (b) polycrystalline racemic DL-Cystine. The clear difference in the spectroscopic signature demonstrates the fact that most signatures of polycrystalline samples in this low frequency range are dominated by intermolecular rather than intramolecular vibrations. 

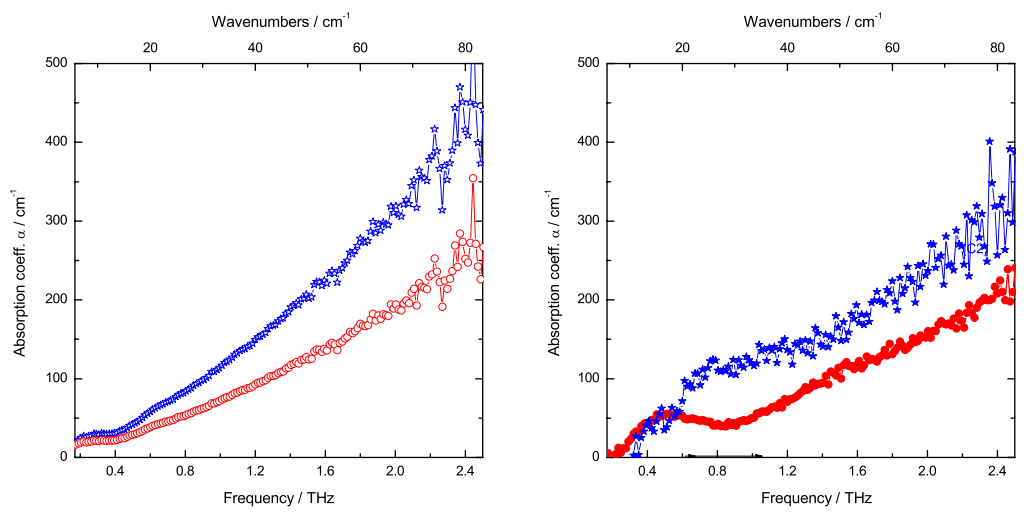

Figure 6. THz absorption spectra of Poly-C (stars) and Poly-A (circles) recorded at ambient temperature. The samples were prepared as pure pressed pellet (left) and as dried, fee-standing films (right).

therefore a combination with other - for example optical - techniques in order to efficiently obtain a maximum of spectroscopic information at different sections of the electromagnetic spectrum with just one sample preparation step seems difficult.

For many biomedical applications, a differentiation between the sample of interest and a reference sample is sufficient. Provided a careful sample preparation, differences in the slope and absolute value of the absorption coefficient can be important parameters to perform such differentiations. For example, we have recently shown that the THz absorption coefficients of two different RNA single strands - polyadenylic acid (Poly-A) and polycydidylic acid (Poly-C) - differ significantly, irrespective of the sample preparation method. ${ }^{17}$ Figure 6 shows the absorption coefficients of these two polynucleotides, prepared as pressed pure pellets (left hand graph) and as dried film (right hand graph). In both cases, Poly-C absorbs stronger as Poly-A. In a next step, we have spotted small drops of Poly-C and Poly-A alternating on a Cycloolefin Homopolymer (Greiner Bio-One) slide, which is transparent for $\mathrm{THz}$ as well as for optical frequencies. The powdery Poly-A and Poly-C samples were diluted in deionized water and then drops containing each $0.2 \mathrm{mg}$ of Poly-A and Poly-C respectively were pipetted by hand onto the slide. Two spots have then been removed in order to identify the orientation of slide in the image. The slide was then placed in the transmission spectrometer and a two dimensional image, shown in figure 7 , was recorded at ambient temperature by moving the sample by steps of $0.5 \mathrm{~mm}$. The Poly-C spots are clearly distinguishable from the Poly-A spots, due to the stronger absorption. A careful check of the spot shape and thickness has been carried out in order to exclude artifacts due to a different thickness. ${ }^{17}$ With this technique, a sensitivity to values below $100 \mu \mathrm{g}$ per spot could be achieved. Although this sensitivity is too low for real clinical applications, it clearly demonstrates the proof of principle. The parameter that was chosen in figure 7 to differentiate between the two polynucleotides is the absolute $\mathrm{THz}$ pulse peak maximum in the time domain, and we have thus not yet fully exploited the complete spectroscopic data. We are therefore confident that by applying advanced signal processing ${ }^{21}$ and novel technical approaches like for example near-field imaging ${ }^{22}$ or differential terahertz time-domain spectroscopy ${ }^{23}$ the sensitivity can be significantly increased.

\section{CONCLUSION}

We have shown that the very characteristic signatures that dominate the spectra of many small, polycrystalline biomolecules allow for contact-free chemical recognition of concealed samples. Although the $\mathrm{THz}$ spectra of larger biomolecles typically show less pronounced features, we have demonstrated that T-ray spectroscopy is a versatile tool for label-free differentiating such complex samples and thus holds a significant potential for biosensing applications. 


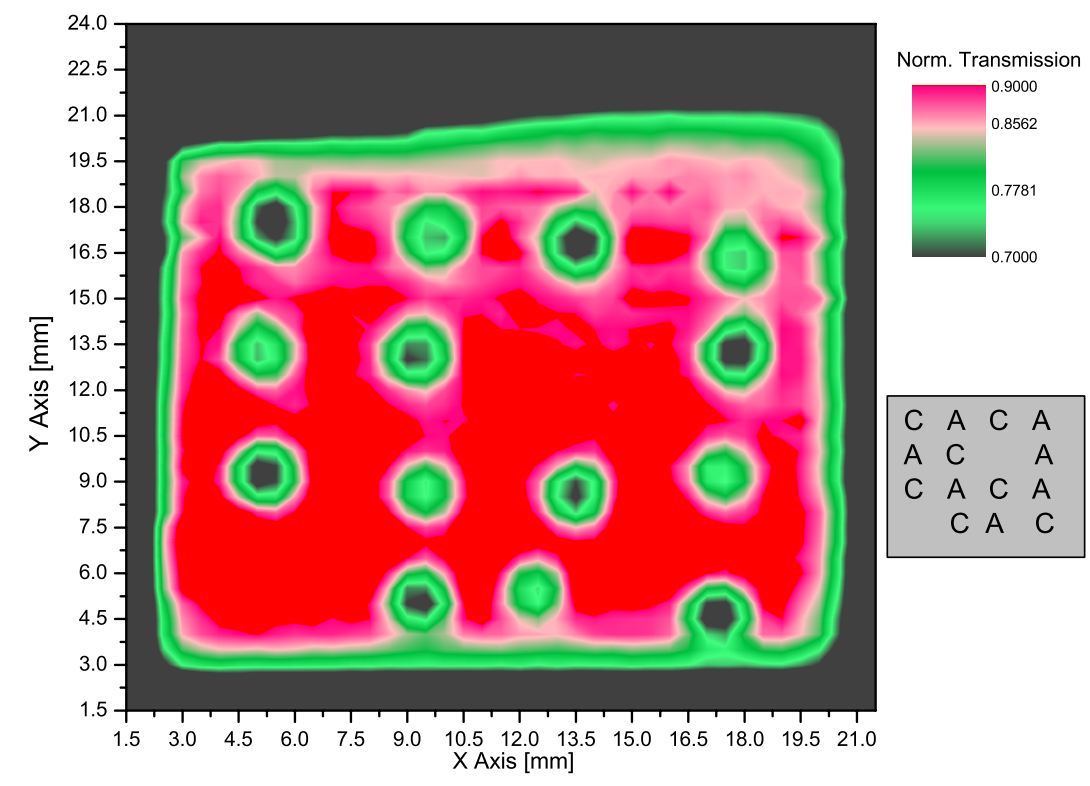

Figure 7. THz image of Poly-A and Poly-C spotted in an alternating pattern (see inset) on a Cycloolefin Homopolymer slide. The colors correspond to the $\mathrm{THz}$ transmission through the sample. The Poly-C spots, appearing darker in the image due to the stronger absorption, can be clearly distinguished from the Poly-A spots.

\section{ACKNOWLEDGEMENT}

The authors would like to Peter Uhd Jepsen for fruitful discussions. We would further like to thank the BMBF, the EU project THz-BRIDGE under contract number QLK4- CT-2000-00129, the Australian Research Council and the Sir Ross and Sir Keith Smith foundation for financial support.

\section{REFERENCES}

1. M. Walther, B. Fischer, M. Schall, H. Helm, and P. U. Jepsen, "Far-infrared vibrational spectra of all-trans, 9-cis and 13-cis retinal measured by THz time-domain spectroscopy," Chem. Phys. Lett. 332, pp. 289-295, 2000 .

2. H.-B. Liu, Y. Chen, and X.-C. Zhang, "Characterisation of anhydrous and hydrated pharmaceutical materials with THz time-domain spectroscopy," Journal of Pharmaceutical Sciences 96(4), pp. 927-934, 2007.

3. J. A. Zeitler, P. F. Taday, D. A. Newnham, M. Pepper, K. C. Gordon, and T. Rades, "Terahertz pulses spectroscopy and imaging in the pharmaceutical setting - a review," Journal of Pharmay and Pharmacology 59(2), pp. 209-223, 2007.

4. R. M. Woodward, "Terahertz technology in biological and chemical sensing for defence," Proceedings of SPIE: Optically Based Biolgical and Chemical Sensing for Defence 5617, pp. 341-352, 2005.

5. H. C. Schau, B. Jenette, C. K. Walker, and C. d'Aubigny, "Design of a low-cost THz imaging scanner," in Proceedings of SPIE: Terahertz for Military and Securiy Applications IV, 6212, art. no. 62120R, 2006.

6. J. J. Strachan, T. Rades, D. A. Newham, K. C. Gordon, M. Pepper, and P. F. Taday, "Using terahertz pulsed spectroscopy to study crystallinity of pharmaceutical materials," Chem. Phys. Letters 390, pp. 20-24, 2004.

7. J. Knab, J. Y. Chen, and A. G. Markelz, "Hydration dependence of confirmational dielectric relaxation of lysozyme," Biophysical Journal 90(7), pp. 2576-2581, 2006. 
8. B. Fischer, M. Hoffmann, H. Helm, G. Modjesch, and P. U. Jepsen, "Chemical recognition in terahertz time-domain spectroscopy and imaging," Semicond. Sci. Technol. 20, pp. 246-253, 2005.

9. P. U. Jepsen, R. Jacobsen, and S. Keiding, "Generation and detection of terahertz pulses from biased semiconductor antennas," J. Opt. Soc. Am. B 13, pp. 2424-2436, 1996.

10. P. U. Jepsen and B. M. Fischer, "Dynamic range in terahertz time-domain transmission and reflection spectroscopy," Optics Letters 30(1), pp. 29-31, 2005.

11. L. Thrane, R. H. Jacobsen, P. U. Jepsen, and S. R. Keiding, "THz reflection spectroscopy of liquid water," Chem. Phys. Lett. 240, p. 330, 1995.

12. B. Fischer, M. Walther, and P. U. Jepsen, "Far-infrared vibrational modes of DNA components studied by terahertz time-domain spectroscopy," Phys. Med.Biol. 47, pp. 3807-3814, 2002.

13. P. C. Uphadya, Y. C. Shen, A. G. Davies, and E. H. Linfield, "Far-Infrared vibrational modes of polycrystalline saccharides," Vibrational Spectroscopy 35, pp. 139-143, 2004.

14. J. F. Federici, B. Schulkin, F. Huang, D. Gary, R. Barat, F. Oliviera, and D. Zimdars, "THz imaging and sensing for security applications - explosives, weapons and drugs," Semicond. Sci. Technol. 20, pp. 266-280, 2005.

15. M. Walther, B. M. Fischer, and P. U. Jepsen, "Noncovalent intermolecular forces in polycrystalline and amorphous saccharides in the far infrared," Chemical Physics 288, pp. 261-268, 2003.

16. M. R. Kuttenruf, C. M. Brown, L. K. Iwaki, M. B. Capbell, T. M. Korter, and E. J. Heilweil, "Terahertz spectroscopy of short-chain polypeptides," Chemical Physics Letters 375, pp. 337-343, 2003.

17. B. M. Fischer, M. Hoffmann, H. Helm, R. Wilk, F. Rutz, T. Kleine-Ostmann, M. Koch, and P. U. Jepsen, "Terahertz time-domain spectroscopy and imaging of artificial RNA," Optics Express 13(14), pp. 5205-5215, 2005.

18. J. Jaques, A. Collet, and S. H. Willen, Enantiomers, Racemates and Resolutions, John Wiley and Sons, Inc., New York, 1981.

19. D. L. Woolard, T. R. Globus, B. L. Gelmont, M. Bykhovskaia, A. C. Samuels, D. Cookmeyer, J. L. Hesler, T. W. Crowe, J. O. Jensen, J. L. Jensen, and W. R. Loerop, "Submillimeter-wave phonon modes in DNA macromolecules," Phys. Rev. E 65(5), art. no. 051903, 2002.

20. M. Nagel, F. Richter, and P. H. B. H. Kurz, "A functionalized THz sensor for marker-free DNA analysis," Phys. Med. Biol. 48, pp. 3625-3636, 2003.

21. X. Yin, B. W.-H. Ng, B. Fischer, B. Ferguson, S. P. Mickan, and D. Abbott, "Feature extraction from terahertz pulses for classification of RNA data via support vector machines," in Proceedings of SPIE: Microand Nanotechnology: Materials, Processes, Packagin and Systems III, 6415, art. no. 641516, 2006.

22. H. Lin, B. M. Fischer, S. P. Mickan, and D. Abbott, "Review of THz near-field methods," in Proceedings of SPIE: Smart Structures, Devices, and Systems III, 6414, art. no. 6414OL, 2007.

23. S. P. Mickan, A. Menikh, H. Liu, C. A. Mannella, R. MacColl, D. Abbott, J. Munch, and X.-C. Zhang, "Label-free bioaffinity detection using terahertz technology," Phys. Med. Biol. 47(21), p. 37893795, 2002. 